\title{
Diet, Nutrition and Modulation of Genomic Expression in Fetal Origins of Adult Disease
}

\author{
Alan A. Jackson ${ }^{a, b}$ Graham C. Burdge ${ }^{a}$ Karen A. Lillycrop ${ }^{b, c}$ \\ anstitute of Human Nutrition, University of Southampton School of Medicine, Southampton General Hospital, \\ ${ }^{b}$ National Institutes of Health Research, Nutrition Biomedical Research Unit, Southampton Universities NHS \\ Trust, Southampton General Hospital, ' Developmental and Cell Biology, University of Southampton School of \\ Biological Sciences, Southampton, UK
}

The greatest burden of ill-health for adults in most societies relates to disorders of the cardiovascular system and to cancers [1]. These diseases are caused by lifestyle choices in which poor diet and relatively low levels of activity play a major role [1]. There is a large body of literature which shows that environmental exposures, including nutrition, play a significant role in the etiology of the disease, but there is variable susceptibility amongst individuals exposed to seemingly similar risk factors or environments. Cancer is a condition which results from a derangement in the cellular processes which regulate cell division, terminal differentiation and apoptosis, with damage to the genetic material of the cell being central. Exposures which lead to these derangements usually precede the appearance of the clinical disease by a prolonged period of time, often several decades [2]. While gene mutation has a role in the etiology of cancer, there is increasing evidence showing that epigenetic processes such as DNA methylation and covalent modification to histones are also involved [3]. Epigenetic changes of this kind represent potential for altered gene activity, and hence cellular dysregulation. However, the underlying propensity may only be manifest when the gene is exposed to an appropriate environmental signal with the direction of the response and nature of the cellular dysregulation a function of the specific epigenetic change $[4,5]$.

It has been known for many years that exposure of the individual to nutritional and other environmental challenges during critical periods of early development can markedly affect later size, shape, structure, function and behavior. However, Barker et al. [6] were the first to provide clear evidence that there might also be a direct link between early nutritional exposure and risk of chronic disease, building the evidence that is supportive of a causal association $[6,7]$. In this they have refined a new conceptual approach to the way in which we consider the evolution of this group of 
diseases based upon a new developmental model, the so called 'fetal origins hypothesis'. Based initially on ecological observations and later on retrospective cohort studies, there is now a considerable body of epidemiological and experimental data that supports the hypothesis. In the earlier observations, the size and shape of the baby at birth was shown to be related to the risk during adult life of coronary heart disease, hypertension, stroke, type 2 diabetes, obesity and some cancers [6]. These relations were shown to be graded across the usual range of birth weights seen in the population and not a special feature of very high or very low birth weight, indicating that they might be a consequence of the usual range of exposure found within a population $[4,7]$. The results have been reproduced across a number of populations, and although there may be some debate around the details, the general principal appears to apply widely $[4,6,7]$.

\section{Epidemiology}

Methodologically, the major advance was to be able to identify groups of adults in whom size at birth had been recorded with reliability and could be related on an individual basis to current health, risk of chronic disease, morbidity or mortality from specific disease conditions [8]. By identifying such populations it has been possible to carry out a range of elegant retrospective cohort investigations providing clear evidence that the pace and pathway of early growth is a major risk factor for this particular group of chronic diseases $[6,8]$. The first studies were carried out in Hertfordshire (England), where it was shown that for both men and women as birth weight increased, the risk of death from coronary heart disease decreased. Based on observations of this kind, the 'fetal origins hypothesis' proposes that poor nutritional exposure during early development of the fertilized ovum, embryo, fetus, infant or child permanently determines the structure and function of the body through the process of programming [7-9]. This translation of genotype into a defined phenotype from a very early stage of development, sets the basis upon which all later exposures, nutritional and those of the wider environment, build. It defines the opportunity and limit for future structure and function [8-10]. More refined exploration of other data sets in which more detailed data are available for later growth during childhood and adolescence, such as the Helsinki Birth Cohort, provide the opportunity to identify particular pathways of growth that may be associated with particular disease outcomes [6]. Together these explorations provide a rich description of how the structural phenotype captured simply as measures of size, shape and body composition mark differences in the functional phenotype which in themselves presage variable vulnerability to a wider range of environmental challenges, which can ultimately lead to ill-health $[6,7]$. These findings from these observational studies are supported by evidence derived from 'natural' experiments. 


\section{Experiments of Nature}

The most well explored human model of a defined intervention for a specific period of time is given by the experience provided as a result of the Dutch famine during 1944-1945. This was a sharply defined period of severe food shortage for a population which previously had enjoyed reasonable nutritional health. During this period the food ration fell below 1,000 kcal/day and even the extra rations allowed for pregnant and lactating women and young children could not be provided $[11,12]$. The change in food availability had a disastrous effect on the population, but although fertility decreased women continued to become pregnant and deliver babies and health records continued to be kept. It has been possible to time the relative exposure of the famine in relation to the time of conception and the progress of the pregnancies. Women who were exposed early in pregnancy, around the time of embryonic growth and the elaboration of the early fetal form had babies which were of relatively good size, but later in life suffered the more extensive and severe manifestations of ill health for a range of systems. Those exposed during later pregnancy, at a time when many of the structures and functions of the fetus have been established, but when the weight gained is greatest, had babies who were most obviously affected by having a lower birth weight. They still carried an increased risk for later ill health, but this was less marked than those exposed during the earlier stages of development. Those fetuses exposed to famine during late gestation were more likely to demonstrate impaired glucose tolerance during adult life. Those exposed in mid-gestation had an increased likelihood of impaired glucose tolerance, but also were more likely to display microalbuminuria indicative of altered renal function or obstructive airways disease indicative of altered respiratory function. Exposure early in gestation was associated with later glucose intolerance, an atherogenic lipid profile, altered blood coagulation, obesity, stress sensitivity, coronary heart disease and breast cancer [11, 12].

These observations demonstrate an effect on a wide range of functions and systems, variability in outcome in relation to the timing of the exposure indicative of special tissue vulnerability at sensitive periods of development, disjunction between the specific functional effects and the size of the baby at birth. In addition to the specific time-related effects from one tissue or system to another, there is also evidence that for those conceived during the famine there was an increased risk of schizophrenia, anti-social personality disorder, and congenital neural defects, demonstrating a wide range of effects on the central nervous system $[11,12]$. The vulnerability implied by increased sensitivity and stress responsiveness increases the potential susceptibility to all stressors and associated metabolic consequences. Importantly, stressors interact with and impact upon nutritional state and wellbeing through a range of effects that include altered appetite, modification in the delivery of nutrients to tissues, altered tissue demands for nutrients and increased nutrient losses. The effects found on liver function (coagulation changes and lipid profiles) and renal health (micralbuminuria) imply the likelihood of altered responsiveness to potential or actual environmental 
toxins, including carcinogens, altered excretion, modified inter-organ co-operativity and integration of function [11, 12]. Not all of the effects should be construed as being necessarily negative. Those women who were exposed to the famine during their own fetal life later showed increased reproductive success [13]. Importantly it has been shown the effects can be passed on to the third generation, arguing strongly for non-genomic transmission of information or memory [14].

Thus, birth size provides some useful information, but has to be seen as a relatively crude indicator of the intrauterine nutritional exposure and experience, patterns of exposure during fetal life set structure and functionality that have long-term impact on the capacity of individual tissues to function and their integration as components of a whole body system, growth and development after birth may be modulated by experience before birth, but how current environmental exposure builds on that experience is itself of importance for later health or disease risk.

\section{Cancer Risk and Early Life}

The fundamental lesion in cancer is damage to DNA, which can be brought about by a range of physical, chemical or microbiological factors. Because the cell usually has mechanisms to protect itself, sustained damage to DNA must take place against a background of a cellular capability that is inadequate to cope by protecting or effectively repairing the damaged DNA. The capability of the cell to achieve effective prevention or repair depends upon the cellular microenvironment, in particular the nutrient microenvironment of the cell. This microenvironment reflects the overall nutritional wellbeing of the individual, a balance between nutrient intake and the specific nutrient demands for usual activity and coping with the rigors imposed by the wider external environment [4]. Cells that contain critically damaged DNA but do not undergo apoptosis are potentially neoplastic. Dysregulation of the life cycle of individual cells is, therefore a fundamental feature of cancer causation. This is in part determined by the immediate nutrient environment, but also by the nutrient environment experienced during very early development $[4,5]$.

The studies from the women who experienced the Dutch hunger winter during early life showed that there was a 5 times increase in risk of developing cancer of the breast in women exposed to famine while in utero [15]. Birth size has been related to increased risk of breast cancer in a number of studies and, in general, larger size at birth is associated with an increased risk of cancer of the breast [2, 5]. In 2007 the World Cancer Research Fund and American Institute of Cancer Research [2] produced the most comprehensive review of any aspect of the medical literature ever conducted. This global effort by some of the world's leading nutrition and cancer scientists identified major factors related to the foods eaten, nutritional status achieved and physical activity undertaken as causal factors for major cancers. This analysis was comprehensive, detailed and rigorous, using sound, validated methods and hence its 
conclusions are the most authoritative statement there is currently on the causes of cancer. It is estimated that on average at least $30 \%$ of cancers could be prevented with appropriate modifications to diet and lifestyle, and in some situations as much as 70\% [16]. There is convincing evidence that abdominal fatness causes colorectal cancer, and probably causes cancer of the pancreas and endometrium and pre-menopausal breast cancer. There is also convincing evidence that achieved height 'causes' colorectal and postmenopausal breast cancer, and probably 'causes' cancer of the pancreas and ovary and premenopausal breast cancer. This very strong relationship between achieved height itself and specific cancer cannot be a direct effect of height on the risk of cancer, but must be a reflection that the complex of factors that contribute to the achievement of height, must also relate strongly, and probably directly to the factors which increase the risk of cancer. Other aspects of development of the bony skeleton have been related to cancer in women in the Helsinki Birth Cohort, where 300 women were diagnosed with breast cancer [6]. Growth of bony skeleton is important for reproductive health in many ways, but most critically during child birth where small pelvic bones increase the risk of obstructed labor. Small pelvic bones are often a persistent consequence of poor nutrition during infancy and childhood. In Helsinki, the dimensions of the mothers' bony pelvis were measured routinely in order to assess the likelihood of an obstructed labor. A higher risk of either breast cancer or ovarian cancer in the daughter was associated with the shape and pattern of the mother's pelvis, itself a marker of the mother's sex hormone status around the time her reproductive capability was being established $[17,18]$. The stem cells for the breast form at around 6 weeks after conception and the authors postulated that higher concentrations of sex hormones in the mother around this time gave rise to genetic instability in the differentiating putative breast cells in their daughters [17]. Further, they found that broader hips in the mother were predictive of ovarian cancer in their daughters, leading to the suggestion that ovarian cancer may be initiated by exposure of the fetal ovary to maternal sex hormones [18].

\section{Growth and Development}

Growth is a complex process that takes place in both space and time. Early exposures can have lasting effects, both on structure and on function. The most obvious example of this is when a noxious exposure acts during a sensitive period of the development of a tissue or organ, impairing the development and leading to lifelong alterations of structure, for example with teratogens such as thalidomide or hypervitaminosis A $[19,20]$. However, a limitation or lack of a specific nutrient can have an equally damaging structural effect, such as hypovitaminosis A, or poor folate status and neural tube defects $[21,22]$. These obvious structural changes at the level of the whole body are extreme forms of less dramatic damage which can be inflicted at the cellular or subcellular levels. 
An understanding of how early nutritional exposure enables normal growth and body proportions, measured indirectly as achieved height and weight, is an imperative if the relationships identified in epidemiological studies of populations are to be interpreted $[7,9,10,23,24]$. Growth is a structured process which includes increases in length and mass, changes in body composition and relative proportions and maturation of function. The elaboration of the processes that enable structure and function at every stage of growth and development result from a complex interaction amongst genetic endowment and the hormonal milieu with the availability of energy and nutrients to fuel and enable cellular elaboration [25]. In an article in the Lancet in 1970, Elsie Widdowson used the term 'harmony of growth' to capture the pace, proportions and partitioning of nutrients that are fundamental for the achievement of normal growth, appropriate body proportions and effective maturation of function [26]. Thus, growth and development are tightly organized and regulated processes with complex and subtle changes taking place in space and time, with each successive change being dependent upon and determined by having achieved the early stages with a measure of success. Any significant constraint at any particular stage of development may lead to alteration of structure and function, which may be difficult or impossible to repair or make good at later times. Any tissue or organ is particularly vulnerable at the time of rapid cellular replication, leading to sensitive periods during development which differ in their critical timing from tissue to tissue or from function to function. Throughout there is a close interdependent relationship between structure and function, thereby capturing a memory of differences in earlier exposure to an altered cellular or tissue, hormonal or nutritional milieu. For any complex organism, this variability in structure and function can obtain at any or every level of organization, extending from the molecular and subcellular, through the organization and regulation of cells, tissues and organs, up to whole body integration of responses to wider environmental challenge. Size at any age is a relatively crude summary statement of the extent to which the availability of energy and pattern of nutrients matches that required for that stage of development, and size at birth is a very crude summary of the nutrients that have been available to the fetus.

The size of newborn babies and their growth during infancy and childhood have changed over time $[27,28]$. During approximately the last 100-150 years, children have been getting larger and growing to maturity more rapidly, known as the secular trend in growth with progressively greater final adult height in many developed countries. Thus, within the same population there has been a progressive increase in attained adult height for both males and females, a reflection of increased height at 5-7 years of age of $1-2 \mathrm{~cm}$ every 10 years $[27,28]$. This has been associated with a decrease in the age of menarche from around 16 to around 13 years of age in the 100 years from 1860 [27]. For a number of countries in western Europe this attained adult height appears to have achieved a plateau of around $1.8 \mathrm{~m}$, for example in Denmark, Sweden, Norway and the Netherlands. It has been suggested that the plateau is achieved around 18 years following post-neonatal mortality falling to around 4/1,000 
deliveries $[23,24]$. In many senses this increase in height is indicative of improvement in public health and much of the increase has been attributed to factors that contribute to improved nutrition from a very early age. Importantly if achieved height is a risk factor for some cancers and there has been a secular trend in height over many years, what are the common factors that underlie this important relationship?

\section{Size and Body Composition at Birth}

The new growth standards developed by WHO show how infants and children should grow when provided with the opportunity for a healthy environment [29]. Across the globe the pattern of growth of children from a wide range of backgrounds is similar. However, even within this similarity for all populations there is variability within the normal range. The suggestion posed by the 'fetal origins hypothesis' is that even within this observed 'normal' range of variability there is differential risk of later chronic disease.

There has been the general observation that infants who are smaller at birth tend to have a different body composition to those who are heavier, most notably that they are relatively more adipose. An extreme example of this difference has been noted in populations where size at birth is very low, for example in India. Yajnick et al. [30] have described the phenotype of the Indian baby as being fat/thin, a pattern that is carried through to adulthood and marks the phenotype which is closely associated with the cardio-metabolic syndrome. Although the baby may be small at birth the relative deficit of different body compartments is not equal. There are substantial deficits in length and lean tissue, but relative preservation of adipose tissue, especially centrally placed adipose. This population has substantially increased risk of type 2 diabetes, associated with relative adiposity throughout life, which may be directly associated with dietary limitations of vitamin $B_{12}$ [31]. One important question is whether this is a peculiarly Indian phenotype and problem, or simply an extreme example of a more common phenotypic difference in size and shape, and also for wider aspects of metabolic function.

Kensara et al. $[32,33]$ compared the extent to which early life variability in size relates to differences in size, shape and whole body function later in life using the Hertfordshire cohort and comparing individuals from the lowest and highest fifths of birth size within the 'normal' range. These men were studied when they were around 70 years old. At this age the percentage body fat was about $5 \%$ greater for those of lower birth weight compared with those of higher birth weight. This meant that for the same weight or BMI at 70 years of age, those of lower birth weight had reduced lean mass, but greater fat mass, especially greater central fat [32]. Resting metabolic rate (RMR) was measured as a summary statement of metabolic activity and $38 \%$ of the variability in RMR could be explained by variation in current size (height and weight) and size at birth. Those in the lower birth weight category had lower resting 
expenditure, $32 \%$ of which was explained by differences in birth size, indicative of a reduced metabolic demand, a reduced metabolic capacity, and altered cellular environment. Size at birth uniquely explained $17 \%$ of the variability, more than current size which uniquely explained $6 \%$ of the variability, with $15 \%$ of the variability explained on a shared basis by size at birth and current height and weight [33]. The compositional changes meant that for any given BMI the men of lower birth weight had 5\% more fat [32]. Therefore, the differences in body composition identified for the Indian baby with the fat/thin phenotype may represent a more general difference in phenotype associated with differences in size at birth, which in itself marks important variability in overall and specific metabolic function and efficiency [34]. Although differences in genotype may explain a part of this variability, the observation that the WHO growth standards apply globally [29] indicate that there are major environmental factors that impact on the variability, which include nutrition factors, either directly or indirectly.

\section{Developmental Plasticity}

The epidemiological and metabolic studies carried out in humans argue strongly that nutrient exposure from the earliest stages of life can exert an impact on functional capability at all later ages, indicating that a single genotype can give rise to a range of different phenotypes. This process, characterized as developmental plasticity, is a widespread biological phenomenon which is considered to enable survival in range of environments, and the ability to cope with the range of stresses or stressors experienced from one situation to another [35]. This variable phenotype may promote the ability to cope in the short term, but carries with it potential vulnerability in the longer term especially if the later environment exposes phenotypic susceptibility. Greater achieved height and central adiposity may confer advantages under some circumstances, but they are not necessarily an unalloyed benefit. It is very difficult to explore these relationships mechanistically in humans, given the long time between the exposure and the outcome, and animal models provide a valuable opportunity to determine possible mechanisms in some detail.

\section{Animal Models}

There are a wide range of studies on the reproductive performance in animals - conducted for the purpose of enhancing animal husbandry - which have explored the effects of general or specific nutritional interventions before or during pregnancy, during lactation and during the later life of the offspring. These generally have an interest in relatively short-term outcomes, determined by market considerations. It is clear that more extreme dietary manipulations lead to adverse outcomes in the 
short and longer term, with the specific consequence being determined by the timing, severity and duration of the insult $[7,19-22]$. An important observation which arises from the epidemiological studies is that for chronic non-communicable disease the variability in risk is seen as a graded effect within the usual variability of birth size and growth within the population. For the diet or nutritional exposure to operate as an important factor in promoting or enabling the altered risk in outcome would require that the effects of importance should be demonstrable across the range of intakes usually seen and considered to be compatible with health within the population. Further, if the impact is cumulative during life it might be explained simply by sensitive periods during development leading to differences in structure and function which constrain the maximal capability of one or other function, or limit the ability to regulate and integrate $[7,25]$. However the observation that these effects can be communicated between generations, and by embryo transfer, requires acquired genetic mechanisms of retained memory, considered most likely to be through epigenetic processes such as DNA methylation and covalent modification of histones [5]. This potentially implicates those processes through which 1-carbon moieties such as methyl groups are made available to metabolism and the mechanisms through which methylation of the promoter region of specific regulatory genes is enhanced or constrained from one situation to another [5].

The induction of changes to the phenotype of the offspring, in response to the prenatal environment, that persists throughout the lifespan implies stable changes to gene transcription resulting in altered activities of metabolic pathways and the set point homeostatic control processes and in differences in the structure of tissues. One important consideration in understanding the mechanism responsible for phenotype induction is the interaction between any process resulting in different phenotypes, environmental cues and gene polymorphisms, in particular those located in gene promoters. Studies on gene expression demonstrate stable effects on transcription [5]. Importantly, some of the genes which showed altered expression following prenatal undernutrition are transcription factors which affect multiple pathways in development and nutrient homeostasis: for example PPAR $\alpha$ and the glucocorticoid receptor (GR) [36]. Modified regulation of expression of a few key transcription factors may alter the activities of a large number of metabolic and developmental pathways. The methylation of $\mathrm{CpG}$ dinucleotides which are clustered at the $5^{\prime}$ promoter regions of genes, confers stable silencing of transcription. Methylation patterns are largely established during embryogenesis or in early postnatal life [37]. DNA methylation also plays a key role in cell differentiation by silencing the expression of specific genes during development and differentiation of individual tissues, and thus the timing of gene methylation is tissue and gene-specific [38,39]. Covalent modifications of histones influence chromatin structure and hence the ability of transcriptional machinery to gain access to DNA. DNA methylation can induce transcriptional silencing by blocking the binding of transcription factors and/or through promoting the binding of the methyl $\mathrm{CpG}$ binding protein (MeCP)-2. The latter binds to methylated cytosines and, 
in turn, recruits histone-modifying complexes composed of deacetylases and histone methyl transferases to the DNA, resulting in a closed chromatin structure and transcriptional silencing $[40,41]$.

Epigenetic regulation of gene promoters is established during development and is responsible for patterns of transcriptional expression and silencing in adults, perturbations to this process represent a candidate molecular mechanism for induction of persistent alterations in phenotype by the environment early in life. Perturbations as diverse as lack of maternal grooming, uterine artery ligation or embryo culture have been shown to lead to epigenetic modulation of transcription, structural and functional effects in the short and long term [5, 42-45].

Varying the maternal intake of nutrients involved in 1-carbon metabolism across a wide range can induce graded changes in DNA methylation and gene expression in the offspring, which persist into adulthood [45]. However, for this mechanism to operate in the induction of phenotypes associated with the 'fetal origins hypothesis' would require that it can operate within the range of dietary intakes typical for a population. Feeding a diet which is adequate but restricted in protein to pregnant rats is a well established model of phenotype induction. This is because feeding pregnant dams graded amounts of protein across a range of intakes not associated with any obvious pathology leads to graded increases in blood pressure in the offspring [46]. This modest change to maternal macronutrient intake during pregnancy induced hypomethylation of the PPAR $\alpha$ and GR promoter and increased expression of PPAR $\alpha$ and GR in the liver of the offspring. There was also an increase in the expression of PPARa and GR target genes such acyl-CoA oxidase and phosphoenolpyruvate carboxykinase, respectively, supporting the suggestion that altered epigenetic regulation of transcription factors modifies that activities of important metabolic pathways [36, 47]. Sequence analysis of the PPAR a promoter showed that the methylation status of only a few CpG dinucleotides was altered by the reduced protein diet during pregnancy [48]. This suggests that the process of induced epigenetic change is targeted and that the resulting change in transcription may reflect changes in the interaction of the gene with relatively few transcription factors, thus inducing specific changes in the regulation of gene function and hence response to environmental differences. Methylation of the PPAR $\alpha$ and GR promoters was also reduced in the heart of animals whose mothers had been exposed to a reduced protein diet during pregnancy [5]. Further, the PPARa promoter was hypomethylated in the whole umbilical cord offspring of rats fed a reduced protein diet during pregnancy [5], suggesting that hypomethylation of PPARa and GR promoters had already been established very early in pregnancy, before cell lineages had become definitively established. Hypomethylation of the GR promoter was associated with an increase in histone modifications which facilitate transcription while those that suppress gene expression were reduced or unchanged [47].

Induction of the altered phenotype (hypertension and endothelial function) in the offspring of rats fed the reduced protein diet during pregnancy was prevented 
by supplementation of this diet with glycine or folic acid [49-51]. Hypomethylation of the hepatic PPARa and GR promoters was also prevented by the addition of 5 times more folic acid than contained in the reduced protein diet [36]. Thus, 1-carbon metabolism plays a central role in the induction of an altered phenotype. In this model there is an important interaction between the metabolism of macronutrients and micronutrients, and further that these interactions operate through differential methylation of the promoter region of regulatory genes through seemingly epigenetic mechanisms. The regulatory genes themselves play a central role in metabolic integration in terms of responsiveness to stress (GR), and macronutrient partitioning and central fat deposition (PPAR $\alpha$ ). Feeding the reduced protein diet during pregnancy in the F0-generation results in elevated blood pressure, endothelial dysfunction, insulin resistance and adverse glucose homeostasis in the F1, F2 and even the F3 generations, despite no further unusual dietary exposure for subsequent generations [52-55]. This implies that transmission of a phenotype induced in the F1 generation to the F2 generation and further into the F3 generation may involve preservation of levels of DNA methylation of specific genes. As the female line appears sufficient for transmission of this epigenetic information between generations the level of methylation of the PPAR $\alpha$ and GR promoters in gametes must be similar to that of somatic cells.

The de novo methylation of CpG dinucleotides is catalyzed by DNA methyltransferase (Dnmt) $3 \mathrm{a}$ and $3 \mathrm{~b}$. The pattern of methylation is maintained through mitosis by gene-specific methylation of hemimethylated DNA by Dnmt1 [37]. Changes in the activity of Dnmt as a result of altered 1-carbon metabolism represent one candidate mechanism for transmission of information regarding maternal 1-carbon metabolism status to the fetus for induction of modified epigenetic regulation of transcription and thus modified phenotype. Feeding the reduced protein diet to rats during pregnancy induced a reduction in Dnmt1 expression and in the binding of Dnmt1 at the GR promoter. [47]. However, the expression of Dnmt3a, Dnmt3b and methyl binding domain-2, and the binding of Dnmt3a at the GR promoter were unaltered [47]. This suggests that hypomethylation of the GR promoter in the liver of the offspring and probably other genes including PPARa, is induced by the maternal diet as a result of a lower capacity to maintain patterns of cytosine methylation during mitosis. Modulation of Dnmt1 expression by differences in 1-carbon metabolism provide a link between maternal diet and epigenetic regulation of gene expression in the fetus. This is supported by the finding that lower Dnmt 1 expression induced by the reduced protein diet during pregnancy was prevented by increasing the folic acid content of the diet. [47] and is consistent with the a central role for Dnmt1 in the induction of an altered phenotype $[49,50]$.

We suggest 2 possible mechanisms by which feeding a reduced protein diet during pregnancy may alter 1-carbon metabolism. Firstly, it is possible that a decreased availability of glycine leads to an altered flux of methyl groups between different metabolic fates and a constraint on the remethylation of homocysteine to methionine [56, 57]. Second, increased maternal corticosteroid levels [58], possibly a result of the stress 
induced by constrained nutrient availability, may reduce folic acid availability [59]. The latter could explain how maternal corticosteroid blockade prevents the induction of hypertension in the offspring of mothers who had reduced protein diets during pregnancy [60], as well as prevention of altered phenotype by folic acid administration $[36,50]$.

Based upon current data, we have suggested a mechanism for the induction of an altered phenotype in the offspring as a consequence of nutrient constraint during pregnancy in which promoter methylation is lost in a gene-specific manner during mitosis due to decreased Dnmt 1 expression and activity $[5,36,61]$. This is accompanied by reduced binding of the MeCP2-histone deacetylase-histone methyltransferase complex leading to persistence of histone modifications that permit transcription.

\section{Epigenetics and Cancer}

A change in the epigenetic regulation of genes has been implicated as a causal mechanism in specific cancers including lung, prostate, breast [62], colon [63] and hemopoietic cancers [64]. Specifically, increased cancer risk is associated with global hypomethylation of the genome with concurrent hypermethylation or hypomethylation of the promoter of specific genes. The mechanism by which global hypomethylation is induced is unclear, but may reflect the global decline in DNA methylation associated with increasing age [62]. The age-related decline in global hypomethylation is related to a reduction in Dnmt1 activity [65] which, in turn, may induce expression of oncogenes such as c-Myc and c-N-ras. [65]. Thus, it appears that modulation of Dnmtl activity is a key regulatory step in both fetal programming and in the induction of tumorigenesis This may be accompanied by de novo methylation of tumor suppressor genes [66] by increased Dnmt3a activity, leading to aberrant activation of genes involved in cell proliferation and cell differentiation [67]. Together these changes represent a shift in the regulation of gene control which, in turn, may predispose the genome to further changes in methylation, which result ultimately in neoplasia.

\section{Conclusion}

The observation that nutrition in early life can induce both hypomethylation and hypermethylation of specific $\mathrm{CpG}$ dinucleotides suggests a mechanism for induction of different disease endpoints (e.g. metabolic disease of cancer) by variation in the same environmental exposure, which is marked by differences in the direction of association between birth weight and disease risk. One particular example of the role of epigenetics in modulating gene activity by shifting the balance between agonist and suppressor proteins is the induction tumorigenesis by activation of telomerase in differentiated cells. Telomerase activity is down-regulated in most cells during terminal 
differentiation in embryogenesis as a result of methylation of the $\mathrm{CpG}$ promoter region. It has been proposed that activation of telomerase in preneoplastic cells is due to a shift in the regulation between the activator $\mathrm{c}-M y c$ and the suppressor WT1, by changes in the methylation status of specific $\mathrm{CpG}$ within the binding domains of these transcription factors in the promoter of the catalytic sub-unit which confers RT activity (hTERT) [68]. One consequence of hTERT activation is to increase Dnmt1 activity [69], which leads to copying of aberrant patterns of cytosine methylation. This suggests a synergistic role for hTERT and Dnmt1 in controlling cell proliferation and the methylation status of the genome.

The addition of supplemental folic acid to the reduced protein diet provided during pregnancy reversed many of the effects of the low protein diet on blood pressure and vascular reactivity as well as on the methylation of the promoter region for PPAR $a$ and GR and their relative expression in the offspring. By contrast when the control protein diet was supplemented with folic acid the effects on the offspring were very different, with an increase in blood pressure and increases in the concentration of triacylglycerol and non-esterified fatty acids in the blood [70]. If the addition of supplemental folic acid to the reduced protein diet provided during pregnancy reversed many of the phenotypic and epigenetic effects of the low protein diet in the offspring would a similar effect be seen if the supplemental folic acid were provided to the offspring postnatally? Supplementation with folic acid postnatally induced increased weight gain, lower plasma $\beta$-hydroxybutyrate concentration and increased hepatic and plasma triacylglycerol concentration compared with offspring not given supplemental folic acid. In the liver of folic acid supplemented offspring there was an increased methylation of the promoter region for PPAR a and the GR, and a decrease in the methylation of the promoter region for the insulin receptor, with reciprocal changes in mRNA expression. Hence increased intakes of supplemental folic acid intake during the juvenile period did not simply reverse the phenotype induced by the maternal diet, but produced distinct changes in both the phenotype and the epigenotype. This indicates that the effect of the increased intake of folic acid is contingent on the timing of the supplementation relative to the developmental stage of the organism and the overall nutrient pattern within the diet. Importantly, whereas during pregnancy the effect of the supplemental folic acid is buffered by maternal metabolism, the juvenile offspring were exposed directly to folic acid provided in the diet [71]. Bidirectional responses in relation to the previous nutritional exposure have also been shown for other systems, for example in rats the expression of $11 \beta$-hydroxysteroid dehydrogenase- 2 in response to leptin administration from day 3 to 10 of life was increased in the offspring of well nourished mothers, but decreased in the offspring of undernourished mothers: by contrast leptin suppressed expression of PPARa in maternally well nourished offspring and enhanced expression in maternally undernourished offspring [72].

The observational evidence from epidemiological studies is substantial. Not surprisingly, not all of the evidence fits in a simple way, but there is a substantial weight of evidence that argues that patterns of growth and development during fetal life, 
infancy and childhood relate strongly to the risk of non-communicable disease during later adult life. The experience drawn from the Dutch winter famine, where there was limited exposure to a very low food intake for a defined period of time, is supportive. Moreover it provides evidence that the timing as well as the severity of the exposure lead to differences in the phenotypic outcome in terms of markers of risk for later ill-health as well as specific disease patterns. Taken together, the evidence argues that the variability in risk cannot be explained simply on the basis of genetic or genomic variability, but appears to be a complex interaction of nutrient exposure and the hormonal milieu at the critical time when tissues and systems are especially sensitive to environmental perturbations, which modifies the opportunity for genetic expression, most likely through epigenetic mechanisms. Animal studies show that modest manipulations of the maternal diet during pregnancy can lead to epigenetic changes in the promoter region of critical regulatory genes, which are carried through generations. These epigenetic changes lead directly to differential expression of the genes and a shift in the set point and responsiveness of regulatory systems. It appears that it is this shift that alters responsiveness of the organism to wider environmental or nutritional perturbations, lowering the threshold for adverse effects and increasing susceptibility to abnormal function. The evidence suggests that aspects of the regulation and control of 1-carbon metabolism are of particular importance in setting the extent of epigenetic modification and, our understanding of the critical factors that determine and control these interactions needs greater refinement. At each age the response to current nutritional exposure appears to be modified to an extent by previous nutritional experience. It appears that metabolic plasticity is directionally dependent on earlier nutritional status and we still do not know what might underlie this response. Population studies show that whereas higher birth weight within an acceptable range is related to better long-term outcome for many of the health issues of concern, such as cardiovascular disease and type 2 diabetes, the opposite is true for cancers such as those of the breast and prostate. If the objective is to move to interventions that will protect the population from ill-health there is an important and urgent need to understand the basis of these different relationships to ensure that the public health implications can be appreciated.

\section{References}

1 World Health Organization: Diet, nutrition and the prevention of chronic disease. Report of as joint WHO/FAO Expert Consultation. WHO Technical report series 916. Geneva, World Health Organization, 2003.

2 World Cancer Research Fund/American Institute for Cancer Research: Food, nutrition, physical activity and the prevention of cancer: a global perspective. Washington, American institute for Cancer Research, 2007.
- 3 Vucic EA, Brown CL, Lam WL: Epigenetics of cancer progression. Pharmacogenomics 2008;9:215234.

4 Jackson AA: Integrating ideas of life course across cellular, individual and population in cancer causation. J Nutr 2005:135(suppl 12):2927S-2933S.

-5 Burdge GC, Lillycrop KA, Jackson AA: Nutrition in early life, and risk of cancer and metabolic disease: alternative endings in an epigenetic tale? Br J Nutr 2009;101: 619-630. 


\section{Journal of
Nutrigenetics utrigenomics}

\begin{tabular}{l|l}
\hline J Nutrigenet Nutrigenomics 2010;3:192-208 \\
\hline $\begin{array}{l}\text { DOI: 10.1159/000324356 } \\
\text { Published online: April 6, 2011 }\end{array}$ & $\begin{array}{l}\text { @ } 2011 \text { S. Karger AG, Basel } \\
\text { www.karger.com/jnn }\end{array}$ \\
\hline
\end{tabular}

Jackson et al.: Diet, Nutrition and Genomic Expression in Fetal Origins of Adult Disease
-6 Barker DJP, Osmond C, Kajantie E, Eriksson JG: Growth and chronic disease: findings in the Helsinki Birth Cohort. Ann Human Biol 2009;36:445-458.

7 Jackson AA: Nutrients, growth, and the development of programmed metabolic function. Adv Exp Med Biol 2000;478:41-55.

8 Barker DJP: Mothers, babies and health in later life, ed 2. Edinburgh, Churchill Livingstone, 1998.

9 Jackson AA: All that glitters. Br Nutr Foundation Nutr Bull 2001;25:11-24.

10 Wootton SA, Jackson AA: Influence of undernutrition in early life on growth, body composition and metabolic competence; in Henry CJ (ed): Early Environment and Later Outcomes. Society for the Study of Human Biology Symposium. Cambridge, Cambridge University Press, 1995.

-11 Painter RC, Roseboom TJ, Bleker OP: Prenatal exposure to the Dutch famine and disease in later life: an overview. Reprod Toxicol 2005;20:345-352.

12 Roseboom T, de Rooij S, Painter R: The Dutch famine and its long-term consequence for adult health. Early Human Dev 2006;82:485-491.

$\checkmark 13$ Painter RC, Westendorp RG, de Rooij SR, et al: Increased reproductive success of women after prenatal undernutrition. Hum Reprod 2008;23:25912595.

14 Painter RC, Osmond C, Gluckman P, et al: Transgenerational effects of prenatal exposure to the Dutch famine on neonatal adiposity and health in later life. BJOG 2008;115:1243-1249.

15 Painter RC, De Rooij SR, Bossuyt PM, et al: A possible link between prenatal exposure to famine and breast cancer: a preliminary study. Am J Hum Biol 2006;18:853-856.

16 World Cancer Research Fund/American Institute for Cancer Research: Policy and action for cancer prevention. Food, nutrition, and physical activity: a global perspective. Washington, AICR, 2009.

17 Barker DJ, Osmond C, Thornburg KL, et al: A possible link between the pubertal growth of girls and breast cancer in their daughters. Am J Hum Biol 2008;20:127-131.

18 Barker DJ, Osmond C, Thornburg KL, Kajantie E, Eriksson JG: A possible link between the pubertal growth of girls and ovarian cancer in their daughters. Am J Hum Biol 2008;20:659-662.

19 Kalter H, Warkany J: Experimental production of congenital malformations in strains of inbred mice by maternal treatment with hypervitaminosis A. Am J Pathol 1961;38:1-21.

20 Woollam DH: Thalidomide disaster considered as an experiment in mammalian teratology. Br Med J 1962;2:236-237.
Wilson JG, Roth CB, Warkany J: An analysis of the syndrome of malformations induced by maternal vitamin A deficiency: effects of restoration of vitamin $\mathrm{A}$ at various times during gestation. Am J Anat 1953;92:189-217.

22 MRC Vitamin Study Research Group: Prevention of neural tube defects: results of the Medical Research Council Vitamin Study. Lancet 1991;338:131-137.

Schmidt IM, Jørgensen MH, Michaelsen KF: Height of conscripts in Europe: is postneonatal mortality a predictor? Ann Hum Biol 1995;22:57-67.

24 Larnkaer A, Attrup Schrøder S, et al: Secular change in adult stature has come to a halt in northern Europe and Italy. Acta Paediatr 2006;95:754-765.

25 Jackson AA: Perinatal nutrition: the impact on postnatal growth and development; in Gluckman PD, Heyman MA (eds): Pediatrics and perinatology, ed 2. London, Arnold, 1996, p 298-303.

26 Widdowson EM: Harmony of growth. Lancet 1970;1:902-905.

27 Tanner JM: Fetus into Man: Physical Growth from Conception to Maturity. Cambridge (Mass.), Harvard University Press, 1990.

28 Cole TJ: Secular trends in growth. Proc Nutr Soc 2000;59:317-324.

29 de Onis M, Garza C, Onyango AW, Borghi E: Comparison of the WHO Child Growth Standards and the CDC 2000 growth charts. J Nutr 2007;137: 144-148.

30 Yajnik CS, Fall CH, Coyaji KJ, et al: Neonatal anthropometry: the thin-fat Indian baby. The Pune Maternal Nutrition Study. Int J Obes Relat Metab Disord 2003;27:173-180.

- 31 Yajnik CS, Deshpande SS, Jackson AA, et al: Vitamin $\mathrm{B}_{12}$ and folate concentrations during pregnancy and insulin resistance in the offspring: the Pune Maternal Nutrition Study. Diabetologia 2008;51:29_ 38.

32 Kensara OA, Wootton SA, Phillips DI, et al: Fetal programming of body composition: relation between birth weight and body composition measured with dual-energy X-ray absorptiometry and anthropometric methods in older Englishmen. Am J Clin Nutr 2005;82:980-987.

- 33 Kensara OA, Wooton SA, Phillips DI, et al: Substrate-energy metabolism and metabolic risk factors for cardiovascular disease in relation to fetal growth and adult body composition. Am J Physiol Endocrinol Metab 2006;291:E365-E371.

34 Sachdev HS, Fall CH, Osmond C, et al: Anthropometric indicators of body composition in young adults: relation to size at birth and serial measurements of body mass index in childhood in the New Delhi birth cohort. Am J Clin Nutr 2005; 82:456-466. 


\section{Nutrigenetics Nutrigenomics}

\begin{tabular}{l|l}
\hline J Nutrigenet Nutrigenomics 2010;3:192-208 \\
\hline DOI: 10.1159/000324356 & $\begin{array}{l}\text { @ } 2011 \text { S. Karger AG, Basel } \\
\text { www.karger.com/jnn }\end{array}$ \\
\hline Published online: April 6, 2011
\end{tabular}

Jackson et al.: Diet, Nutrition and Genomic Expression in Fetal Origins of Adult Disease
- 35 Bateson P, Barker D, Clutton-Brock T, et al: Developmental plasticity and human health. Nature 2004;430:419-421.

-36 Lillycrop KA, Phillips ES, Jackson AA, Hanson MA, Burdge GC: Dietary protein restriction of pregnant rats induces and folic acid supplementation prevents epigenetic modification of hepatic gene expression in the offspring. J Nutr 2005; 135:13821386.

- 37 Bird A: DNA methylation patterns and epigenetic memory. Genes Dev 2002;16:6-21.

- 38 Gidekel S, Bergman Y: A unique developmental pattern of Oct-3/4 DNA methylation is controlled by a cis-demodification element. J Biol Chem 2002;277: 34521-34530.

- 39 Hershko AY, Kafri T, Fainsod A, Razin A: Methylation of HoxA5 and HoxB5 and its relevance to expression during mouse development. Gene 2003:302;65-72.

40 Fuks F, Hurd PJ, Wolf D, et al: The methyl-CpGbinding protein $\mathrm{MeCP} 2$ links DNA methylation to histone methylation. J Biol Chem 2003;278:40354040.

-41 Turner BM: Histone acetylation and an epigenetic code. Bioessays 2000;22:836-845.

$\checkmark 42$ Weaver IC, Cervoni N, Champagne FA, et al: Epigenetic programming by maternal behavior. Nat Neurosci 2004;7:847-854.

-43 Szyf M, Weaver I, Meaney M: Maternal care, the epigenome and phenotypic differences in behavior. Reprod Toxicol 2007;24:9-19.

44 Pham TD, MacLennan NK, Chiu CT, et al: Uteroplacental insufficiency increases apoptosis and alters p53 gene methylation in the full-term IUGR rat kidney. Am J Physiol Regul Integr Comp Physiol 2003;285:R962-R970.

-45 Waterland RA, Jirtle RL: Early nutrition, epigenetic changes at transposons and imprinted genes, and enhanced susceptibility to adult chronic diseases. Nutrition 2004;20:63-68.

-46 Langley SC, Jackson AA: Increased systolic blood pressure in adult rats induced by fetal exposure to 57 maternal low protein diets. Clin Sci (Lond) 1994;86: 217-222.

-47 Lillycrop KA, Slater-Jefferies JL, Hanson MA, et al: Induction of altered epigenetic regulation of the hepatic glucocorticoid receptor in the offspring of rats fed a protein-restricted diet during pregnancy suggests that reduced DNA methyltransferase-1 expression is involved in impaired DNA methylation and changes in histone modifications. Br J Nutr 2007;97:1064-1073
48 Lillycrop KA, Phillips ES, Torrens C, et al: Feeding pregnant rats a protein-restricted diet persistently alters the methylation of specific cytosines in the hepatic PPAR alpha promoter of the offspring. $\mathrm{Br} \mathrm{J}$ Nutr 2008;100:278-282.

49 Jackson AA, Dunn RL, Marchand MC, LangleyEvans SC: Increased systolic blood pressure in rats induced by a maternal low-protein diet is reversed by dietary supplementation with glycine. Clin Sci (Lond) 2002;103:633-639.

50 Brawley L, Torrens C, Anthony FW, et al: Glycine rectifies vascular dysfunction induced by dietary protein imbalance during pregnancy. J Physiol 2004; 554:497-504.

51 Torrens C, Brawley L, Anthony FW, et al: Folate supplementation during pregnancy improves offspring cardiovascular dysfunction induced by protein restriction. Hypertension 2006;47:982-987.

-52 Martin JF, Johnston CS, Han CT, Benyshek DC: Nutritional origins of insulin resistance: a rat model for diabetes-prone human populations. J Nutr 2000; 130:741-744.

-53 Zambrano E, Martínez-Samayoa PM, Bautista CJ, et al: Sex differences in transgenerational alterations of growth and metabolism in progeny (F2) of female offspring (F1) of rats fed a low protein diet during pregnancy and lactation. J Physiol 2005;566:225236.

54 Benyshek DC, Johnston CS, Martin JF: Glucose metabolism is altered in the adequately-nourished grand-offspring (F3 generation) of rats malnourished during gestation and perinatal life. Diabetologia 2006;49:1117-1119.

55 Torrens C, Poston L, Hanson MA: Transmission of raised blood pressure and endothelial dysfunction to the F2 generation induced by maternal protein restriction in the $\mathrm{F} 0$, in the absence of dietary challenge in the F1 generation. Br J Nutr 2008;100:760766.

56 Jackson AA: The glycine story. Eur J Clin Nutr 1991;45:59-65.

7 Meakins TS, Persaud C, Jackson AA: Dietary supplementation with L-methionine impairs the utilization of urea-nitrogen and increases 5-L-oxoprolinuria in normal women consuming a low protein diet. J Nutr 1998:128;720-772.

58 Langley-Evans SC, Gardner DS, Jackson AA: Maternal protein restriction influences the programming of the rat hypothalamic-pituitary-adrenal axis. J Nutr 1996;126:1578-1585.

59 Terzolo M, Allasino B, Bosio S, et al: Hyperhomocysteinemia in patients with Cushing's syndrome. J Clin Endocrinol Metab 2004;89:3745-3751. 


\section{Nutrigenetics \\ Nutrigenomics}

\begin{tabular}{l|l}
\hline J Nutrigenet Nutrigenomics 2010;3:192-208 \\
\hline $\begin{array}{l}\text { DOI: 10.1159/000324356 } \\
\text { Published online: April 6, } 2011\end{array}$ & $\begin{array}{l}\text { ○ } 2011 \text { S. Karger AG, Basel } \\
\text { www.karger.com/jnn }\end{array}$ \\
\hline
\end{tabular}

Jackson et al.: Diet, Nutrition and Genomic Expression in Fetal Origins of Adult Disease
60 Langley-Evans SC: Hypertension induced by foetal exposure to a maternal low-protein diet, in the rat, is prevented by pharmacological blockade of maternal glucocorticoid synthesis. J Hypertens 1997;15: 537-544.

-61 Burdge GC, Slater-Jefferies J, Torrens C, et al: Dietary protein restriction of pregnant rats in the F0 generation induces altered methylation of hepatic gene promoters in the adult male offspring in the F1 and F2 generations. Br J Nutr 2007;97:435-439.

-62 Liu L, Wylie RC, Andrews LG, Tollefsbol TO: Aging, cancer and nutrition: the DNA methylation connection. Mech Ageing Dev 2003;124:989-998.

63 Zhu J: DNA methylation and hepatocellular carcinoma. J Hepatobiliary Pancreat Surg 2006;13:265273.

64 Galm O, Herman JG, Baylin SB: The fundamental role of epigenetics in hematopoietic malignancies. Blood Rev 2006;20:1-13.

65 Lopatina N, Haskell JF, Andrews LG, et al: Differential maintenance and de novo methylating activity by three DNA methyltransferases in aging and immortalized fibroblasts. J Cell Biochem 2002; 84:324-334.

66 Lengauer C: Cancer: an unstable liaison. Science 2003;300:442-443.
67 Strathdee G, Appleton K, Illand M, et al: Primary ovarian carcinomas display multiple methylator phenotypes involving known tumor suppressor genes. Am J Pathol 2001;158:1121-1127.

68 Tollefsbol TO, Andrews LG: Mechanisms for telomerase gene control in aging cells and tumorigenesis. Med Hypotheses 2001;56:630-637.

69 Young JI, Sedivy JM, Smith JR: Telomerase expression in normal human fibroblasts stabilizes DNA 5-methylcytosine transferase I. J Biol Chem 2003; 278:19904-19908.

70 Burdge GC, Lillycrop KA, Jackson AA, Gluckman PD, Hanson MA: The nature of the growth pattern and of the metabolic response to fasting in the rat are dependent upon the dietary protein and folic acid intakes of their pregnant dams and post-weaning fat consumption. Br J Nutr 2008;99:540-549.

71 Burdge GC, Lillycrop KA, Phillips ES, et al: Folic acid supplementation during the juvenile-pubertal period in rats modifies the phenotype and epigenotype induced by prenatal nutrition. J Nutr 2009; 139:1054-1060.

72 Gluckman PD, Lillycrop KA, Vickers $\mathrm{MH}$, et al: Metabolic plasticity during mammalian development is directionally dependent on early nutritional status. Proc Natl Acad Sci USA 2007;104:1279612800.

Prof. Alan A. Jackson

Institute of Human Nutrition, Southampton General Hospital (MP 113) 